Pacific

Journal of

Mathematics

THREE REMARKS ON MEAN FIELD EQUATIONS

LI MA 


\title{
THREE REMARKS ON MEAN FIELD EQUATIONS
}

\author{
LI MA
}

\begin{abstract}
We prove the Liouville theorem for the mean field equation (also called the conformal curvature equation) in $\mathbb{R}^{2}$, an a priori bound for solutions of the mean field equation on the negative part of indefinite nonlinearity, and the symmetry property of mean field equation on an annulus with zero Dirichlet boundary condition.
\end{abstract}

We study the mean field equation (also called the conformal curvature equation) in a smooth domain $D$ of the plane $\mathbb{R}^{2}$; that is, we study

$$
-\Delta u=K(x) e^{u} \quad \text { for } x \in D,
$$

where $K=K(x)$ is a smooth function on $\bar{D}$. Usually $K(x)$ is assumed to be positive, but here we allow $K(x) e^{u}$ to change sign; then (1) is said to have indefinite nonlinearity. This equation has received much attention in recent years for its rich physical and geometrical content, such as its relation with the Nirenberg problem on $S^{2}$ in geometry and with Chern-Simons-Higgs theory [Yang 2001] in gauge theory. See [Cheng and Ni 1991] and [Lin 2007] for deep results on the mean field equations and related topics. See also [Ma and Wei 2001] and [Tarantello 2004].

Using the boundary blow-up method [Du and Ma 2001], we have the following Liouville theorem for the Liouville equation on $\mathbb{R}^{2}$; see [Liouville 1853].

Theorem 1. Let $H(x)$ be a positive smooth function on $\mathbb{R}^{2}$. Assume that there are positive constants $C>0, R_{0}>0$, and $\beta \in[0,2)$ such that $\inf _{|x| \leq R} H(x) \geq C R^{-\beta}$ for all $R>R_{0}$. Then there is no $u \in C^{2}\left(\mathbb{R}^{2}\right)$ satisfying the Liouville equation

$$
\Delta u=H(x) e^{u} \quad \text { for } x=\left(x_{1}, x_{2}\right) \in \mathbb{R}^{2} .
$$

When $H(x)=1$, this result with the extra assumption $\int_{\mathbb{R}^{2}} e^{u}<\infty$ was proved in [Li 1999] by the spherical averaging method. Actually, this result can be easily derived from [Osserman 1957] and [Keller 1957]. We present it here because it

MSC2000: primary 35J60; secondary 53C21, 58J05.

Keywords: mean field equation, conformal curvature, indefinite nonlinearity, moving sphere method, Liouville theorem.

This research is partially supported by the National Natural Science Foundation of China, grant number 10631020, and SRFDP 20060003002. 
is useful in local estimates with indefinite nonlinearity. Using the method used to prove Theorem 1, we have this:

Theorem 2. Let $u$ be a solution of (1) with $D=B_{R}(0)$. Assume that there is a positive constant $C_{0}>0$ such that $K(x) \leq-C_{0}$ in the ball $B_{R}(0)$. Then there is a constant $C=C\left(R, \sup _{B_{R}} K\right)$ such that $u(x) \leq C$ in $B_{R / 2}(0)$.

Following the classic paper [Gidas et al. 1979], Chen and Li [2008] proved this result on $S^{2}$ by the moving plane method. Our method is simpler.

Let $a>1$ be a fixed constant. Let $A=\left\{x \in \mathbb{R}^{2}: a^{-1}<|x|<a\right\}$ be an annulus. Using the moving sphere method as in [Chen and Li 1995; Padilla 1997], we have:

Theorem 3. For $x \in \mathbb{R}^{2}$, let $r=|x|$. Suppose the function $K(x)$ of (1) is continuous in $\bar{A}$ and satisfies $K(x)<0$ for $r<1$, and $K\left(\lambda^{2} x / r^{2}\right)-K(x)<0$ for every $\lambda>1$ and $r>\lambda$. Assume $u \in C^{2}(A) \cap C(\bar{A})$ satisfies (1) in A with the Dirichlet boundary condition $u=0$ on $\partial A$.

Then

$$
u(x) \geq u\left(x / r^{2}\right)-4 \log r \quad \text { for all points } x \text { satisfying } 1 \leq|x| \leq a,
$$

and either

$$
\partial_{r} u(z)=-2 /|z| \text { for some point } z \text { satisfying } 1<|z|<a,
$$

or else

$$
\partial_{r} u(x)>-2 / r \text { for all points } x \text { satisfying } 1<|x|<a .
$$

In the proof of Theorem 3, we may use the notation $u=u(r)$ since the angular variable plays no role.

Padilla [1997] used a similar method to show radial symmetry of solutions for other nonlinear Dirichlet problems on higher-dimensional annuli.

Proof of Theorem 1. Choose any fixed point $x_{0} \in \mathbb{R}^{2}$, and let $R>0$. Let $r=\left|x-x_{0}\right|$ and let $\epsilon>0$. Define

$$
M(x):=M_{R, \epsilon}(x)=2 \log \frac{2 R}{\sqrt{\epsilon}\left(\mathbb{R}^{2}-r^{2}\right)} \quad \text { for } r \leq R .
$$

The metric $g=e^{M(x)} d x^{2}$ on $B_{R}=B_{R}\left(x_{0}\right)$ is the Poincaré metric with scalar curvature $-2 \epsilon$. Hence $\Delta M=2 \epsilon e^{M}$ for $x \in B_{R}$ and $M(x) \rightarrow \infty$ as $x \rightarrow \partial B_{R}$. Choose $\epsilon=C R^{-\beta} / 2$, where $R>R_{0}$. By our assumption on $H$, we have $\Delta u \geq 2 \epsilon e^{u}$ in $B_{R}$.

Let $w=u-M$. Note that $\Delta w \geq 2 \epsilon\left(e^{u}-e^{M}\right)=C(x) w$ for $x \in B_{R}$, where $C(x)=2 \epsilon\left(e^{u}-e^{M}\right) /(u-M)>0$. Note that $w(x) \rightarrow-\infty$ as $x \rightarrow \partial B_{R}$. Hence by maximum principle we have $w \leq 0$ in $B_{R}$. Then we have

$$
u\left(x_{0}\right) \leq M\left(x_{0}\right)=2 \log \frac{2}{\sqrt{\epsilon} R} \rightarrow-\infty \quad \text { as } R \rightarrow \infty,
$$


which contradicts that $u\left(x_{0}\right)$ is finite.

Proof of Theorem 2. Without loss of generality we may let $C_{0}=1$. Recall that $\Delta u=(-K) e^{u} \geq e^{u}$ in $B_{R}$. Let $w=u-M_{R, 1}$. Then as in our previous proof, $\Delta w \geq C(x) w$ for $x \in B_{R}$. Then by maximum principle again, we have $w(x) \leq 0$ for $x \in B_{R}$, that is, $u(x) \leq M_{R, 1}(x)$ for $x \in B_{R}$. Hence

$$
u(x) \leq 2 \log (8 /(3 R)) \quad \text { for } x \in B_{R / 2} .
$$

This result is a local version of one obtained in [Chen and Li 2008] by the moving sphere method, which we will now use.

Proof of Theorem 3. Given $\lambda \in(1, a)$, let $T_{\lambda}=\partial B_{\lambda}$ and $\Sigma_{\lambda}=\left\{x \in \mathbb{R}^{2}: \lambda<|x|<a\right\}$.

For $x \in \Sigma_{\lambda}$, let $x^{\lambda}=\lambda^{2} x /|x|^{2}$. Note that

$$
|x|>\left|x^{\lambda}\right|=\lambda^{2} /|x|>1 /|x| \text { on } \Sigma_{\lambda} .
$$

Recall that the Kelvin transform $v(x)$ for the function $u(x)$ outside the unit ball $B:=B_{1}(0) \subset \mathbb{R}^{2}$ is $v(x)=u\left(x /|x|^{2}\right)-4 \log |x|$.

For any $\lambda>0$, we define

$$
v_{\lambda}(x)=u\left(\lambda^{2} x /|x|^{2}\right)+4 \log \lambda-4 \log |x| .
$$

Noting that $\Delta\left(u\left(\lambda^{2} x /|x|^{2}\right)\right)=\left(\lambda^{4} /|x|^{4}\right) \Delta u\left(\lambda^{2} x /|x|^{2}\right)$, we have

$$
-\Delta v_{\lambda}=K\left(\lambda^{2} x /|x|^{2}\right) e^{v_{\lambda}} .
$$

Let $w_{\lambda}(x)=u(x)-v_{\lambda}(x)$ on $\Sigma_{\lambda}$. Then we have

$$
-\Delta w_{\lambda}=K\left(\lambda^{2} x /|x|^{2}\right) C(x, \lambda) w_{\lambda}+\left(K(x)-K\left(\lambda^{2} x /|x|^{2}\right)\right) e^{u} \quad \text { on } \Sigma_{\lambda},
$$

where

$$
C(x, \lambda):=\left(e^{u(x)}-e^{v_{\lambda}(x)}\right) /\left(u(x)-v_{\lambda}(x)\right)>0 .
$$

By assumption, we have $\left(K\left(\lambda^{2} x /|x|^{2}\right)-K(x)\right) e^{u} \leq 0$ on $\Sigma_{\lambda}$, and then

$$
-\Delta w_{\lambda} \geq K\left(\lambda^{2} x /|x|^{2}\right) C(x, \lambda) w_{\lambda} \quad \text { on } \Sigma_{\lambda} .
$$

Note that $w_{\lambda}=0$ on $T_{\lambda}$. We remark that for $\lambda=1$, we have $w_{1} \geq 0$ on $\partial \Sigma_{1}$. By the maximum principle and Hopf's boundary point lemma, we have $w_{1}>0$ in $\Sigma_{1}$, which is (3), and $\partial_{r} w_{1}>0$ on $|x|=1$. We now assume that (4) is not true.

We claim that $\partial_{r} w_{\lambda}>0$ on $T_{\lambda}$ for all $1<\lambda<a$.

This claim is true for $\lambda$ near 1 by continuity. Then by using the standard moving sphere method and Hopf's boundary point lemma, we need only to show that for $\lambda \in[1, a)$, there is a neighborhood $U_{\lambda}$ of $T_{\lambda}$ in $\Sigma_{\lambda}$ such that

$$
w_{\lambda}>0 \text { in } U_{\lambda} \text { and } \partial_{r} w_{\lambda}>0 \text { on } T_{\lambda} .
$$


and that this neighborhood depends continuously on $\lambda$. In fact, let

$$
\lambda_{1}=\sup \left\{\lambda \in[1, a): w_{\tilde{\lambda}}>0 \text { for all } \tilde{\lambda} \leq \lambda \text { and } x \in U_{\tilde{\lambda}}\right\} .
$$

If $\lambda_{1}<a$, by continuity, we have $w_{\lambda_{1}} \geq 0$ in $T_{\lambda_{1}}$ and $\left.\partial_{r} w_{\lambda_{1}}\right|_{r=\lambda_{1}} \geq 0$ in $T_{\lambda_{1}}$. By our assumption that (4) is not true, we must have $\left.\partial_{r} w_{\lambda_{1}}\right|_{r=\lambda_{1}}>0$ in $T_{\lambda_{1}}$. By this, we conclude that there is some $\epsilon>0$ and a neighborhood $U_{\lambda}$ of $T_{\lambda}$ in $\Sigma_{\lambda}$ such that $w_{\lambda}>0$ in $U_{\lambda}$ for all $\lambda \in\left[\lambda_{1}, \lambda_{1}+\epsilon\right)$; this contradicts the definition of $\lambda_{1}$.

Hence, $\lambda_{1}=a$. Setting $r=\lambda>1$ in the second inequality of (7), we have

$$
\partial_{r} u(r)>-2 / r \text { on } \Sigma_{1} .
$$

This is the desired inequality (5).

\section{Acknowledgment}

I would like to thank the referee for pointing out some misprints in the previous version of the paper.

\section{References}

[Chen and Li 1995] W. X. Chen and C. M. Li, "A necessary and sufficient condition for the Nirenberg problem”, Comm. Pure Appl. Math. 48:6 (1995), 657-667. MR 96j:35056 Zbl 0830.35034

[Chen and Li 2008] W. X. Chen and C. M. Li, "A priori estimate for the Nirenberg problem", Discrete Contin. Dyn. Syst. Ser. S 1:2 (2008), 225-233. MR 2009c:35108 Zbl 1156.35025

[Cheng and Ni 1991] K.-S. Cheng and W.-M. Ni, "On the structure of the conformal Gaussian curvature equation on $\mathbb{R}^{2}$, II”, Math. Ann. 290:4 (1991), 671-680. MR 92i:53017 Zbl 0753.35032

[Du and Ma 2001] Y. Du and L. Ma, "Logistic type equations on $\mathbb{R}^{N}$ by a squeezing method involving boundary blow-up solutions", J. London Math. Soc. (2) 64:1 (2001), 107-124. MR 2002d:35089 Zbl 1018.35045

[Gidas et al. 1979] B. Gidas, W.-M. Ni, and L. Nirenberg, "Symmetry and related properties via the maximum principle”, Comm. Math. Phys. 68:3 (1979), 209-243. MR 80h:35043 Zbl 0425.35020

[Keller 1957] J. B. Keller, "On solutions of $\Delta u=f(u)$ ", Comm. Pure Appl. Math. 10 (1957), 503510. MR 19,964c Zbl 0090.31801

[Li 1999] Y. Y. Li, "Harnack type inequality: The method of moving planes", Comm. Math. Phys. 200:2 (1999), 421-444. MR 2000c:58024 Zbl 0928.35057

[Lin 2007] C.-S. Lin, "An expository survey on the recent development of mean field equations", Discrete Contin. Dyn. Syst. 19:2 (2007), 387-410. MR 2008f:35123 Zbl 1159.35025

[Liouville 1853] J. Liouville, "Sur l'equation aux derivatives partielles $\partial^{2} \log \lambda / \partial u \partial v \pm \lambda / 2 a^{2}=0$ ", J. Math. Pure Appl. 18 (1853), 71-74.

[Ma and Wei 2001] L. Ma and J. C. Wei, "Convergence for a Liouville equation”, Comment. Math. Helv. 76:3 (2001), 506-514. MR 2002h:35097 Zbl 0987.35056

[Osserman 1957] R. Osserman, "On the inequality $\Delta u \geq f(u)$ ", Pacific J. Math. 7 (1957), 16411647. MR 20 \#4701 Zbl 0083.09402

[Padilla 1997] P. Padilla, "Symmetry properties of positive solutions of elliptic equations on symmetric domains”, Appl. Anal. 64:1-2 (1997), 153-169. MR 98b:35067 Zbl 0942.35084 
[Tarantello 2004] G. Tarantello, "Analytical aspects of Liouville-type equations with singular sources", pp. 491-592 in Stationary partial differential equations, I, edited by M. Chipot and P. Quittner, North-Holland, Amsterdam, 2004. MR 2006e:35124 Zbl 1129.35408

[Yang 2001] Y. Yang, Solitons in field theory and nonlinear analysis, Springer, New York, 2001. MR 2002m:58001 Zbl 0982.35003

Received September 3, 2008. Revised February 1, 2009.

\section{MA}

Department of Mathematical SCiEnces

TSINGHUA UNIVERSITY

BEIJING 100084

CHINA

1ma@math.tsinghua.edu.cn 